AEI-2000-037

hep-th/0006234

\title{
Bulk Witten Indices and the Number of Normalizable Ground States in Supersymmetric Quantum Mechanics of Orthogonal, Symplectic and Exceptional Groups
}

\author{
Matthias Staudacher * \\ Albert-Einstein-Institut, Max-Planck-Institut für Gravitationsphysik \\ Am Mühlenberg 1 \\ D-14476 Golm, Germany
}

\begin{abstract}
This note addresses the question of the number of normalizable vacuum states in supersymmetric quantum mechanics with sixteen supercharges and arbitrary semi-simple compact gauge group, up to rank three. After evaluating certain contour integrals obtained by appropriately adapting BRST deformation techniques we propose novel rational values for the bulk indices. Our results demonstrate that an asymptotic method for obtaining the boundary contribution to the index, originally due to Green and Gutperle, fails for groups other than $\mathrm{SU}(N)$. We then obtain likely values for the number of ground states of these systems. In the case of orthogonal and symplectic groups our finding is consistent with recent conjectures of Kac and Smilga, but appears to contradict their result in the case of the exceptional group $G_{2}$.
\end{abstract}

*matthias@aei-potsdam.mpg.de 
Supersymmetric Yang-Mills theories dimensionally reduced to zero spacial dimensions were initially considered as interesting examples for susy quantum mechanics [1], [2]. The Hamiltonian of these systems reads

$$
H=\frac{1}{2 g^{2}} \operatorname{Tr}\left(P_{i} P_{i}-\frac{1}{2}\left[X_{i}, X_{j}\right]\left[X_{i}, X_{j}\right]-\Psi_{\alpha}\left[\Gamma_{\alpha \beta}^{i} X_{i}, \Psi_{\beta}\right]\right) .
$$

where the bosonic $\left(X_{i}\right)$ and fermionic $\left(\Psi_{i}\right)$ degrees of freedom take values in the Lie algebra of the compact gauge group. Due to the representation theory of the gamma matrices $\Gamma_{\alpha \beta}^{i}$ this quantum mechanics only exists if the number of supercharges is $\mathcal{N}=2,4,8$ or 16 , corresponding to the dimensional reduction of $\mathcal{N}=1$ supersymmetric gauge field theory in $D=d+1$ dimensions, where $d=2,3,5,9$, respectively (i.e. $i=1, \ldots, d$ ).

The $d=9$ system gained relevance following work of de Wit, Hoppe and Nicolai [3], who argued that the light cone quantization of 11-dimensional supermembranes could be described by the model in eq.(1) with gauge group $\mathrm{SU}(N)$ in the limit $N \rightarrow \infty$. This interpretation rendered more urgent the question about the Hamiltonian's spectrum. It was quickly understood that the latter is continuous and, in fact, that there are non-localized states for any positive energy eigenvalue [2], [0]. As far as the supermembrane is concerned, this was initially considered to be an unphysical feature. More recently, the model was "resuscitated" as a proposed formulation of M-theory [5], albeit for a special background, and, interestingly, the continuous spectrum was turned into a virtue. A crucial issue, required in all known applications of the $d=9$ system eq.(1), is whether there also exists a normalizable zero energy vacuum state (which may loosely be called the "graviton multiplet"). On the other hand, from various points of view, one does not expect such a state in the cases $d=2,3,5$. A rigorous proof for $d=3,5,9$ of this has so far only been proposed for $\mathrm{SU}(2)$ [7] (see also [6]). For alternative insights into this question see [8], [9], [10].

For the group $G=\mathrm{SU}(N)$, the proof has not yet been fully completed, but a strategy generalizing the method of [6], [7], as well as some important partial results, exists. In fact, the procedure immediately applies to more general compact gauge groups $G$. We will exclude from now on discussion of the case $d=2$, where the methods below appear to fail. The idea is to compute the Witten index ind ${ }^{D=d+1}(G)$ of the quantum mechanics eq.(间):

$$
\text { ind }^{D}(G)=\lim _{\beta \rightarrow \infty} \operatorname{Tr}(-1)^{F} e^{-\beta H}=n_{B}^{0}-n_{F}^{0}
$$

giving the number of $n_{B}^{0}$ bosonic minus $n_{F}^{0}$ fermionic zero energy states. Clearly ind ${ }^{D}(G)$ gives a lower bound on the number of vacuum states. This lower bound has recently been argued to be saturated for the systems we are studying [11. In light of the above we would

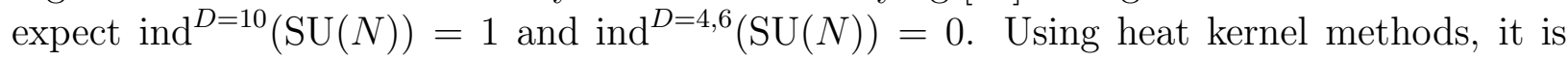
technically much easier to calculate the so-called bulk index

$$
\operatorname{ind}_{0}^{D}(G)=\lim _{\beta \rightarrow 0} \operatorname{Tr}(-1)^{F} e^{-\beta H}
$$

which may be related to a finite susy Yang-Mills integral $\mathcal{Z}_{D, G}^{\mathcal{N}}$ :

$$
\operatorname{ind}_{0}^{D}(G)=\frac{1}{\mathcal{F}_{G}} \mathcal{Z}_{D, G}^{\mathcal{N}}
$$

This is an ordinary (as opposed to functional) multiple integral given by 


$$
\mathcal{Z}_{D, G}^{\mathcal{N}}:=\int \prod_{A=1}^{\operatorname{dim}(G)}\left(\prod_{\mu=1}^{D} \frac{d X_{\mu}^{A}}{\sqrt{2 \pi}}\right)\left(\prod_{\alpha=1}^{\mathcal{N}} d \Psi_{\alpha}^{A}\right) \exp \left[\frac{1}{4 g^{2}} \operatorname{Tr}\left[X_{\mu}, X_{\nu}\right]\left[X_{\mu}, X_{\nu}\right]+\frac{1}{2 g^{2}} \operatorname{Tr} \Psi_{\alpha}\left[\Gamma_{\alpha \beta}^{\mu} X_{\mu}, \Psi_{\beta}\right]\right]
$$

where $\operatorname{dim}(G)$ is the dimension of the Lie group and the $D=d+1$ bosonic matrices $X_{\mu}=X_{\mu}^{A} T^{A}$ and the $\mathcal{N}$ fermionic matrices $\Psi_{\alpha}=\Psi_{\alpha}^{A} T_{A}$ are anti-hermitean and take values in the fundamental representation of the Lie algebra $\operatorname{Lie}(G)$, whose generators we denote by $T^{A} \cdot g^{2}$ is fixed according to the normalization $\operatorname{Tr} T^{A} T^{B}=-g^{2} \delta^{A B}$. The constant $\mathcal{F}_{G}$ in eq.(画) is essentially the volume of the true gauge group, which turns out to be the quotient group $G / Z_{G}$, with $Z_{G}$ the center group of $G$. For more details see [17].

The integral eq.(5) is still very complicated and has so far only been directly analytically calculated for $G=\mathrm{SU}(2)$ [2], [6], [7]. It has however been indirectly calculated for $\mathrm{SU}(N)$ by supersymmetric BRST deformation techniques in 12. The derivation involved some assumptions and unproven steps, but the result has been confirmed for various values of $N$ and $D$ in numerical (Monte Carlo) studies [13]. The result may be summarized in the following table.

Table 1: $D=4, D=6$ and $D=10$ bulk index $\operatorname{ind}_{0}^{D}$ and (for $N>2$, conjectured) total Witten index $\operatorname{ind}^{D}$ for the special unitary groups of arbitrary rank.

\begin{tabular}{||c||c|c|c|c|c|}
\hline Group & rank & $\operatorname{ind}_{0}^{D=4,6}$ & $\operatorname{ind}^{D=4,6}$ & $\operatorname{ind}_{0}^{D=10}$ & $\operatorname{ind}^{D=10}$ \\
\hline \hline $\mathrm{SU}(\mathrm{N})$ & $N-1$ & $1 / N^{2}$ & 0 & $\sum_{m \mid N} 1 / m^{2}$ & 1 \\
\hline
\end{tabular}

We see that ind ${ }^{D} \neq \operatorname{ind}_{0}^{D}$ contrary to what one might have suspected: The continous spectrum renders $\operatorname{Tr}(-1)^{F} e^{-\beta H} \beta$-dependent, and the full Witten index $\operatorname{ind}^{D}$ is given by

$$
\operatorname{ind}^{D}(G)=\operatorname{ind}_{0}^{D}(G)+\operatorname{ind}_{1}^{D}(G) .
$$

where $\operatorname{ind}_{1}^{D}$ is a "boundary term" that is picked up when going from eq.(2) to eq.(3). Its value has been rigorously evaluated for $\mathrm{SU}(2)$ in [7]. For general special unitary groups, it has been argued by Green and Gutperle [14 that the boundary term may be deduced by considering a free effective Hamiltonian for the diagonal (Cartan) degrees of freedom of the $d$ Lie-algebra valued fields $X_{i}$, with the discrete Weyl symmetry of $G$ imposed as a constraint on the wave functions. This led to the evaluation of a simple Gaussian integral and in consequence to

$$
\operatorname{ind}_{1}^{D=4,6}(\mathrm{SU}(N))=-\frac{1}{N^{2}} \quad \text { and } \quad \operatorname{ind}_{1}^{D=10}(\mathrm{SU}(N))=-\sum_{\substack{m \mid N \\ m>1}} \frac{1}{m^{2}} .
$$

Incidentally, the $D=10$ expression was proposed before the evaluation of the bulk indices [14. It seems therefore very likely that the numbers of table 1 are indeed correct for all $N$. In order to further test these ideas, it is clearly of interest to apply them to various other gauge groups. As we shall see, most of the features just discussed become more intricate.

The zero-energy bound state problem for the Hamiltonian eq.(1) for general semi-simple gauge groups was recently considered in detail by Kac and Smilga [15]. Generalizing the mass 
deformation method of [10], these authors made the very interesting claim that the YangMills quantum mechanics can lead for $D=10$ to more than one vacuum state. Assuming that all large mass bound states remain normalizable as the mass is tuned to zero - this is the potential weak point of the method - they found

$$
\begin{aligned}
& \text { ind }^{D=10}(\mathrm{SO}(N))=\text { number of partitions of } N \text { into distinct odd parts } \\
& \text { ind }^{D=10}(\mathrm{Sp}(2 N))=\text { number of partitions of } 2 N \text { into distinct even parts } \\
& \text { ind }^{D=10}\left(\mathrm{G}_{2}\right)=2 \quad \operatorname{ind}^{D=10}\left(\mathrm{~F}_{4}\right)=4 \\
& \operatorname{ind}^{D=10}\left(\mathrm{E}_{6}\right)=3 \quad \operatorname{ind}^{D=10}\left(\mathrm{E}_{7}\right)=6 \quad \operatorname{ind}^{D=10}\left(\mathrm{E}_{8}\right)=11
\end{aligned}
$$

In the case of the $\mathrm{SO}(N)$ and $\operatorname{Sp}(2 N)$, Hanany et.al. presented independent arguments in favor of these multiplicities by considering a "physical" application of the $d=9$ Yang-Mills quantum mechanics to orientifold points in $M$ theory [16].

Clearly it is worthwhile to apply the index computations of the last section to the new classes of groups. In fact, Kac and Smilga [15] generalized the Green-Gutperle [14] method based on a free effective Hamiltonian, and, after imposing the discrete Weyl symmetry and performing the appropriate Gaussain integration, proposed for $D=4,6$ the explicit formula

$$
\operatorname{ind}_{1}^{D=4,6}(G)=?-\frac{1}{\left|W_{G}\right|} \sum_{w \in W_{G}}^{\prime} \frac{1}{\operatorname{det}(1-w)}
$$

where the sum $\sum^{\prime}$ extends over all elements $w$ of the Weyl group $W_{G}$ of $G$ such that $\operatorname{det}(1-w) \neq 0$. They also derived a slightly more involved $D=10$ expression. For $G=\mathrm{SU}(N)$ one quickly rederives eq.(7) from these expressions.

One should next compute the bulk indices $\operatorname{ind}_{0}^{D}(G)$. It is straightforward, in principle, to extend the deformation method of Moore et.al. [12] to the new groups. This was done in [17 for $D=4$ and rank up to three. We carefully evaluated the corresponding (see eq.(画) Yang-Mills integrals eq.(5) by Monte Carlo and verified that the BRST deformation method indeed applies to the new groups as well. Surprisingly, the result did not match the expression eq.(9) (remember that we expect $\operatorname{ind}_{0}^{D=4}+\operatorname{ind}_{1}^{D=4}=0$ ). This indicates failure of the description based on an effective free Hamiltonian.

In the present note we will apply the BRST deformation method [12 to also work out the bulk indices of the $D=10$ model. This method consists in adding cubic and quadratic terms to the action which break all but one of the supersymmetries. The remaining symmetry still assures that the partition function remains unchanged. By taking limits the non-linearities of the original action are simply dropped, and all integrations can be performed. For $D=10$ one finds the $r$-fold integral

$$
\begin{gathered}
\operatorname{ind}_{0}^{D=10}(G)=\frac{\left|Z_{G}\right|}{\left|W_{G}\right|} C^{r} \oint \prod_{k=1}^{r} \frac{d x_{k}}{2 \pi i} \Delta_{G}(0, x) \frac{\Delta_{G}\left(E_{1}+E_{2}, x\right) \Delta_{G}\left(E_{1}+E_{3}, x\right) \Delta_{G}\left(E_{2}+E_{3}, x\right)}{\Delta_{G}\left(E_{1}, x\right) \Delta_{G}\left(E_{2}, x\right) \Delta_{G}\left(E_{3}, x\right) \Delta_{G}\left(E_{4}, x\right)} \\
C=\frac{\left(E_{1}+E_{2}\right)\left(E_{1}+E_{3}\right)\left(E_{2}+E_{3}\right)}{E_{1}+E_{2}+E_{3}+E_{4}}
\end{gathered}
$$


where $\left|Z_{G}\right|$ and $\left|W_{G}\right|$ are the orders of, respectively, the center group $Z_{G}$ and Weyl group $W_{G}$ of $G$, and $r$ is the rank of $G$. For the various groups one has (see [17 for details):

$$
\begin{gathered}
\Delta_{\mathrm{SO}(2 N+1)}(E, x)=\prod_{i<j}^{N}\left[\left(x_{i}-x_{j}\right)^{2}-E^{2}\right]\left[\left(x_{i}+x_{j}\right)^{2}-E^{2}\right] \prod_{i=1}^{N}\left[x_{i}^{2}-E^{2}\right] \\
\Delta_{\mathrm{Sp}(2 N)}(E, x)=\prod_{i<j}^{N}\left[\left(x_{i}-x_{j}\right)^{2}-E^{2}\right]\left[\left(x_{i}+x_{j}\right)^{2}-E^{2}\right] \prod_{i=1}^{N}\left[x_{i}^{2}-\left(\frac{E}{2}\right)^{2}\right] \\
\Delta_{\mathrm{SO}(2 N)}(E, x)=\prod_{i<j}^{N}\left[\left(x_{i}-x_{j}\right)^{2}-E^{2}\right]\left[\left(x_{i}+x_{j}\right)^{2}-E^{2}\right] \\
\Delta_{\mathrm{G}_{2}}(E, x)=\left[\left(x_{1}-x_{2}\right)^{2}-E^{2}\right]\left[\left(x_{1}+x_{2}\right)^{2}-E^{2}\right]\left[x_{1}^{2}-E^{2}\right]\left[x_{2}^{2}-E^{2}\right]\left[\left(2 x_{1}+x_{2}\right)^{2}-E^{2}\right]\left[\left(x_{1}+2 x_{2}\right)^{2}-E^{2}\right]
\end{gathered}
$$

The integrals eq.(10 are divergent as ordinary integrals over $\mathbb{R}^{r}$; if however one (a) interprets them as contour integrals, and (b) gives a small imaginary part to the real parameters $E_{j} \rightarrow E_{j}+i \epsilon$ they converge. The resulting number is, after imposing $\operatorname{Re}\left(E_{4}\right)=-\operatorname{Re}\left(E_{1}\right)-$ $\operatorname{Re}\left(E_{2}\right)-\operatorname{Re}\left(E_{3}\right)$, independent of the parameters $E_{j}$. As we already remarked, the above presciptions would have to be derived from first principles if one wants to render the BRST deformation method entirely rigorous, but we have good evidence [13], [17] that it really works for $D=4,6,10$.

It would be interesting to evaluate the $D=4,6,10$ contour integrals for general $\mathrm{SO}(2 N+$ 1), $\mathrm{Sp}(2 N)$ and $\mathrm{SO}(2 N)$, as has been done (at least for $D=4,6$ ) for $\mathrm{SU}(N)$ in [12. In particular, it would be exciting to find the correct replacement for eq.(9). This has to date proved too difficult; what can be done is an evaluation for small rank. For $D=10$ even this is rather involved, and we needed to apply symbolic manipulation programs to locate and evaluate the huge number of residues for the contour integrals eq.(10). The results, for rank up to three, are shown in the table 2 below.

We would next like to be able to find the total index $\operatorname{ind}^{D=10}(G)$ in order to verify the conjectures eq.(8). As we have demonstrated already for $D=4,6$, and may be verified for $D=10$ as well by comparing the numbers in table 2 with those of [15], we are currently lacking a reliable method for determining the boundary index $\operatorname{ind}_{1}^{D=10}(G)$. Given only the bulk index $\operatorname{ind}_{0}^{D=10}(G)$, can we still make a statement about the number of vacuum states? In all known cases the boundary index is a negative rational number between zero and one. Assuming this to be true in general, we are able to predict the Witten index, which has to be an integer. This prediction is shown in the rightmost column of table 2. Comparing

\footnotetext{
${ }^{1}$ This is actually not entirely true. If the $E_{j}$ are rational numbers there are numerous "special" configurations where simple poles merge to higher order poles in the denominator of the integrand and the integral gives a wrong value. The correct bulk index is only obtained for a "generic" pole configuration.
} 
to eq.(8), we find agreement for the orthogonal and symplectic groups of rank $\leq 3$ (in particular: ind ${ }^{D=10}(\operatorname{Sp}(6)=2)$, but disagreement for the exceptional group $\mathrm{G}_{2}$. This means that either the mass deformation method [10], [15] fails in this case, or that the boundary term is a positive number. It is clearly and exciting problem to resolve this puzzle.

Table 2: $D=4, D=6$ and $D=10$ bulk index $\operatorname{ind}_{0}^{D}$ and (conjectured) total Witten index ind $^{D}$ for the orthogonal, symplectic and exceptional groups of rank $\leq 3$. Previously unknown values are printed in bold-face.

\begin{tabular}{||c||c|c|c|c|c|}
\hline Group & rank & $\operatorname{ind}_{0}^{D=4,6}$ & ind $^{D=4,6}$ & ind $_{0}^{D=10}$ & ind $^{D=10}$ \\
\hline \hline $\mathrm{SO}(3)$ & 1 & $1 / 4$ & 0 & $5 / 4$ & 1 \\
$\mathrm{SO}(4)$ & 2 & $1 / 16$ & 0 & $25 / 16$ & 1 \\
$\mathrm{SO}(5)$ & 2 & $9 / 64$ & 0 & $\mathbf{8 1 / 6 4}$ & 1 \\
$\mathrm{SO}(6)$ & 3 & $1 / 16$ & 0 & $21 / 16$ & 1 \\
$\mathrm{SO}(7)$ & 3 & $25 / 256$ & 0 & $\mathbf{3 2 5 / 2 5 6}$ & 1 \\
\hline \hline $\mathrm{Sp}(2)$ & 1 & $1 / 4$ & 0 & $5 / 4$ & 1 \\
$\mathrm{Sp}(4)$ & 2 & $9 / 64$ & 0 & $\mathbf{8 1 / 6 4}$ & 1 \\
$\mathrm{Sp}(6)$ & 3 & $51 / 512$ & 0 & $\mathbf{1 1 7 5 / 5 1 2}$ & 2 \\
\hline \hline $\mathrm{G}_{2}$ & 2 & $151 / 864$ & 0 & $\mathbf{1 3 7 5 / 8 6 4}$ & $\mathbf{1} ?$ \\
\hline
\end{tabular}

In summary, we have demonstrated that Witten index calculations for supersymmetric gauge quantum mechanics remain very subtle and are fraught with pitfalls. In particular the available methods for computing the boundary contribution to the index appear to be unreliable. We furthermore found some evidence that mass deformation methods might fail for some gauge groups as well. On the other hand, the BRST deformation method for the bulk index appears to be fully valid in $D=4,6,10$. It would be exciting to compute the resulting contour integrals for a general gauge group. For $D=10$ it should be interesting to further check the method by numerical techniques. Finally, it will be important to rigorously derive the proposed number of $D=10$ vacuum states, in particular in the controversial case of the exceptional groups.

\section{ACKNOWLEDGMENTS}

We thank W. Krauth and A. Smilga for useful discussions. 


\section{REFERENCES}

[1] M. Claudson and M. Halpern, Supersymmetric Ground State Wave Functions, Nucl. Phys. B250 (1985) 689; M. Baake, M. Reinecke and V. Rittenberg, Fierz Identities for Real Clifford Algebras and the Number of Supercharges, J. Math. Phys. 26 (1985) 1070; R. Flume, On Quantum Mechanics with Extended Supersymmetry and Non-abelian Gauge Constraints, Annals Phys. 164 (1985) 189.

[2] A.V. Smilga, Witten Index Calculation in Supersymmetric Gauge Theory, Yad. Fiz. 42 (1985) 728, Nucl. Phys. B266 (1986) 45; A.V. Smilga, Calculation of the Witten Index in Extended Supersymmetric Yang-Mills Theory, (in Russian) Yad. Fiz. 43 (1986) 215.

[3] B. de Wit, J. Hoppe and H. Nicolai, On the Quantum Mechanics of Supermembranes, Nucl. Phys. B305 (1988) 545.

[4] B. de Wit, M. Lüscher and H. Nicolai, The supermembrane is unstable, Nucl. Phys. B320 (1989) 135.

[5] T. Banks, W. Fischler, S. Shenker and L. Susskind, M-theory as a Matrix Model: A Conjecture, Phys. Rev. D55 (1997) 5112, hep-th/9610043.

[6] P. Yi, Witten Index and Threshold Bound States of D-Branes, Nucl. Phys. B505 (1997) 307, hep-th/9704098.

[7] S. Sethi and M. Stern, D-Brane Bound State Redux, Commun. Math. Phys. 194 (1998) 675 , hep-th/9705046.

[8] J. Hoppe, Zero Energy States in Supersymmetric Matrix Models, Class. Quant. Grav. 17 (2000) 1101, and references therein.

[9] M. Bordemann and J. Hoppe, Zero Energy States for SU(N): A Simple Exercise in Group Theory?, hep-th/9909191; J. Hoppe, Asymptotic Zero Energy States for $S U(N \geq 3)$ hep-th/9912163; J. Hoppe and J. Plefka, The Asymptotic Ground State of SU(3) Matrix Theory, hep-th/0002107.

[10] M. Porrati and A. Rozenberg, Bound States at Threshold in Supersymmetric Quantum Mechanics, Nucl. Phys. B515 (1998) 184, hep-th/9708119.

[11] S. Sethi and M. Stern, Invariance Theorems for Supersymmetric Yang-Mills Theories, hep-th/0001189.

[12] G. Moore, N. Nekrasov and S. Shatashvili, D-particle bound states and generalized instantons, Commun. Math. Phys. 209 (2000) 77, hep-th/9803265.

[13] W. Krauth, H. Nicolai and M. Staudacher, Monte Carlo Approach to M-Theory, Phys. Lett. B431 (1998) 31, hep-th/9803117; W. Krauth and M. Staudacher, Finite Yang-Mills Integrals, Phys. Lett. B435 (1998) 350, hep-th/9804199; W. Krauth and M. Staudacher, Eigenvalue Distributions in Yang-Mills Integrals, Phys. Lett. B453 (1999) 253, hep-th/9902113.

[14] M. B. Green and M. Gutperle, D-Particle Bound States and the D-Instanton Measure, JHEP 9801 (1998) 005, hep-th/9711107.

[15] V.G. Kac and A.V. Smilga, Normalized Vacuum States in $\mathcal{N}=4$ Supersymmetric Yang-Mills Quantum Mechanics with any Gauge Group, Nucl. Phys. B571 (2000) 515, hep-th/9908096.

[16] A. Hanany, B. Kol and A. Rajaraman, Orientifold Points in M Theory, JHEP 9910 (1999) 027, hep-th/9909028.

[17] W. Krauth and M. Staudacher, Yang-Mills Integrals for Orthogonal, Symplectic and Exceptional Groups, accepted for publication in Nuc. Phys. B, hep-th/0004076. 